\title{
Multipoint connectivity analysis of the May 2007 solar energetic particle events
}

\author{
E. E. Chollet, ${ }^{1}$ R. A. Mewaldt, ${ }^{1}$ A. C. Cummings, ${ }^{1}$ J. T. Gosling, ${ }^{2}$ D. K. Haggerty, ${ }^{3}$ \\ Q. Hu, ${ }^{4}$ D. Larson, ${ }^{5}$ B. Lavraud,,${ }^{6,7}$ R. A. Leske, ${ }^{1}$ A. Opitz, ${ }^{6,7}$ E. C. Roelof, ${ }^{3}$ C. T. Russell, ${ }^{8}$ \\ and J.-A. Sauvaud ${ }^{6,7}$
}

Received 12 April 2010; revised 14 September 2010; accepted 30 September 2010; published 11 December 2010.

[1] In May of 2007, the STEREO Ahead and Behind spacecraft, along with the ACE spacecraft situated between the two STEREO spacecraft, observed two small solar energetic particle (SEP) events. STEREO-A and -B observed nearly identical time profiles in the 19 May event, but in the 23 May event, the protons arrived significantly earlier at STEREO-A than at STEREO-B and the time-intensity profiles were markedly different. We present SEP anisotropy, suprathermal electron pitch angle and solar wind data to demonstrate distortion in the magnetic field topology produced by the passage of multiple interplanetary coronal mass ejections on 22 and 23 May, causing the two spacecraft to magnetically connect to different points back at the Sun. This pair of events illustrates the power of multipoint observations in detailed interpretation of complex events, since only a small shift in observer location results in different magnetic field line connections and different SEP time-intensity profiles.

Citation: Chollet, E. E., et al. (2010), Multipoint connectivity analysis of the May 2007 solar energetic particle events, J. Geophys. Res., 115, A12106, doi:10.1029/2010JA015552.

\section{Introduction}

[2] The acceleration locations, acceleration mechanisms and transport of energetic particles remain major mysteries in astrophysics, and solar energetic particles (SEPs) in the interplanetary medium provide one of the best laboratories for testing energetic particle theories. Most of the largest SEP events seem to originate from bow shocks produced by fast coronal mass ejections (CMEs), which are ejected from the Sun with speeds up to $\sim 3000 \mathrm{~km} / \mathrm{s}$. Though some particles are accelerated far from the Sun, comparisons of coronal images with the arrival times of SEPs at Earth indicate that the first SEPs are released close to the Sun, when the shock has reached $\sim 3-10$ solar radii $\left(\mathrm{R}_{\odot}\right)$ [see, e.g., Kahler, 1994; Tylka et al., 2003; Mewaldt et al., 2003; Reames,

\footnotetext{
${ }^{1}$ Space Radiation Laboratory, California Institute of Technology, Pasadena, California, USA.

${ }^{2}$ Laboratory for Atmospheric and Space Physics, University of Colorado at Boulder, Boulder, Colorado, USA.

${ }^{3}$ Applied Physics Laboratory, Johns Hopkins University, Laurel, Maryland, USA.

${ }^{4}$ Center for Space Plasma and Aeronomy Research, University of Alabama in Huntsville, Huntsville, Alabama, USA.

${ }^{5}$ Space Sciences Laboratory, University of California, Berkeley, California, USA.

${ }^{6}$ Centre d'Etude Spatiale des Rayonnements, Université de Toulouse, Toulouse, France.

${ }^{7}$ Centre National de la Recherche Scientifique, UMR 5187, Toulouse, France.

${ }^{8}$ Institute of Geophysics and Planetary Physics, University of California, Los Angeles, California, USA.

Copyright 2010 by the American Geophysical Union. 0148-0227/10/2010JA015552
}

2009]. The particles then stream out through the solar system, following magnetic field lines. If the shock is broad, it can accelerate particles over field lines in a large solid angle, and SEPs can be quickly distributed over a wide range of longitudes where they can be observed by observers separated by 90 degrees or more. Although the time-intensity profiles of the resulting SEP events are observed to be quite different by spacecraft separated by many tens of degrees in latitude [e.g., Lario et al., 2006; Kallenrode, 1993], observers within a few degrees of each other usually see very similar profiles [von Rosenvinge et al., 2009].

[3] Single-spacecraft studies have shown that changes in energetic particle arrival times, intensities and anisotropies reflect changes in magnetic field line length or connection [e.g., Saiz et al., 2008; Torsti et al., 2004; Bieber et al., 2005]. For example, an in situ spacecraft observing a mix of field lines connected and not connected to a particle source may see intermittent, dispersionless dropouts in the energetic particle intensity [Mazur et al., 2000; Giacalone et al., 2000]. As a spacecraft crosses a boundary in the solar wind, such as the heliospheric current sheet or the outer edge of an interplanetary coronal mass ejection (ICME), the point at the Sun to which the field line connects can shift many tens of degrees, resulting in dropouts in both ions and electrons [Gosling et al., 2004a] as well as changes in the low-energy heat flux of electrons [Gosling et al., 2004b]. Most of the studies cited here were performed on flare-related energetic particle events, which typically have much smaller source regions than CME-related events, so connection shifts at the Sun producing changes in energetic particle time-intensity profiles are observed frequently, in about half of events [Chollet and Giacalone, 2008]. However, in theory, if the 


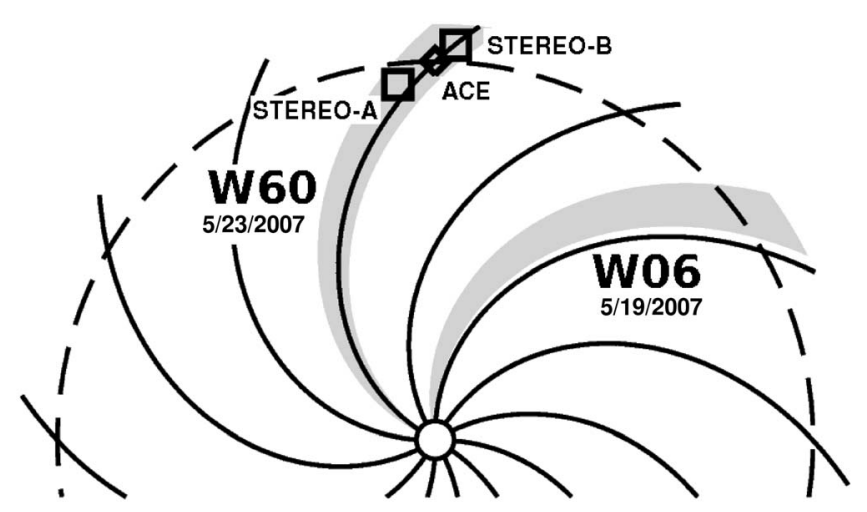

Figure 1. The positions of the two STEREO spacecraft (squares) and ACE (diamond) during May 2007. The centers of the nominal Parker spiral connections to the flare regions at W06 and W60 are shown in gray for a $400 \mathrm{~km} / \mathrm{s}$ solar wind.

field lines observed by spacecraft were to trace back to locations with significantly more or less particle acceleration, the spacecraft should observe changes in the timeintensity profiles of the energetic particles even in large CME-related events.

[4] One of the prime objectives of the Solar Terrestrial Relations Observatory (STEREO [Kaiser et al., 2008]) mission is to perform multipoint in situ measurements of SEPs, which give insight into the geometry of these SEP events not available from single spacecraft observations. By comparing the sudden intensity changes in two closely separated spacecraft, we can determine whether they are connected to the same or different parts of the particle acceleration region at the Sun. The magnetic topology of the inner heliosphere is often modeled for space weather predictions but rarely sampled directly, making in situ SEP studies of connection changes valuable for comparison with numerical predictions.

[5] In this work, we compare and contrast two SEP events occurring in May 2007. These events were observed by the two STEREO spacecraft as well as the Advanced Composition Explorer (ACE) [Stone et al., 1998], providing a threepoint perspective on the energetic particles and the magnetic connections that control their transport. We show how the passage of flux ropes through near-Earth space during this complex period caused the spacecraft to observe field lines which were connected to very different points at the Sun during the 23 May event. In section 2, we present the observations of these events, and in section 3 we present the interpretation and analysis.

\section{Observations}

[6] The relative geometry of the spacecraft and particle sources is key in determining the magnetic field line connections. During May 2007, the STEREO-Ahead spacecraft was $\sim 6.1^{\circ}$ ahead of the Earth while STEREO-Behind trailed the Earth by $\sim 3.1^{\circ}$. The ACE spacecraft is in a halo orbit around Earth's L1 point, so it was between the two STEREO spacecraft, with STEREO-A roughly $7 \times 10^{6} \mathrm{~km}$ closer to the Sun than ACE and STEREO-B roughly $8 \times 10^{6} \mathrm{~km}$ farther out. On 19 May 2007 (day of year (DOY) 139), AR10956 was at longitude W06, and it produced a class B9.5 flare with an X-ray peak at 1302 UT. According to the SOHO/ LASCO catalog, the $\mathrm{C} 2$ and $\mathrm{C} 3$ coronagraphs observed the launch of a small CME beginning at 1324 UT with a speed of $\sim 1000 \mathrm{~km} / \mathrm{s}$. By 23 May, the same active region had rotated to longitude W60. It produced a class B5.3 flare with an X-ray peak at $0732 \mathrm{UT}$ and a SOHO/LASCO CME with a speed of $\sim 650 \mathrm{~km} / \mathrm{s}$. Figure 1 shows the relative positions of these spacecraft, along with the nominal Parker spiral connections to $\mathrm{W} 6$ and $\mathrm{W} 60$ for a $400 \mathrm{~km} / \mathrm{s}$ solar wind. The two spacecraft would have been nominally well connected to the active region by a Parker spiral field for the 23 May event but would have been observing the flank of the 19 May event in a simple Parker spiral field.

[7] This study includes energetic particle, suprathermal electron, plasma and magnetic field data from the twin STEREO spacecraft and from ACE. The STEREO SEP observations reported here were made with the Low-Energy Telescope (LET) [Mewaldt et al., 2008] which measures H and He SEP composition and energy spectra from $\sim 2.5$ to $\sim 15 \mathrm{MeV} /$ nucleon. The LET sensors have a double-ended field of view that covers $\sim 130^{\circ}$ of the ecliptic plane in both the front and rear hemispheres centered at $45^{\circ}$ from the radial, but less than $\pm 20^{\circ}$ in the direction normal to the plane. STEREO measurements of suprathermal electrons are performed by the Solar Wind Electron Analyzer (SWEA) [Sauvaud et al., 2008] and magnetic field measurements by the MAG sensor [Acuña et al., 2008]. These three instruments are part of the IMPACT investigation [Luhmann et al., 2008] on STEREO. The solar wind density, velocity and temperature data are obtained from The Plasma and Suprathermal Ion Composition instruments (PLASTIC) [Galvin et al., 2008].

[8] The ACE energetic ion data come from the Electron, Proton and Alpha Monitor (EPAM) [Gold et al., 1998]. The suprathermal electron data and solar wind data come from the Solar Wind Electron Proton Alpha Monitor (SWEPAM) [McComas et al., 1998] and the Solar Wind Ion Composition Spectrometer (SWICS) [Gloeckler et al., 1998] and magnetic field data from the MAG instrument [Smith et al., 1998]. ACE spins around an axis generally pointed along the Earth-Sun line, and these instruments sample more of the unit sphere out of the ecliptic.

[9] The composition of these events suggests that the particles were accelerated by CME-driven shocks rather than by flares, though there is some evidence for contamination by flare-accelerated particles in the 23 May event. Flareaccelerated SEPs typically show enhancements in the rare species ${ }^{3} \mathrm{He}$ of a factor of $10-1000$ over solar wind values [Mason et al., 2002] along with enhancements in $\alpha$ particles and heavy species like iron [Mason et al., 2004]. In these events, no ${ }^{3} \mathrm{He}$ or iron was detected by either LET instrument $(\sim 2-15 \mathrm{MeV} / \mathrm{nuc})$. At lower energies $(\sim 0.1-2.0 \mathrm{MeV} /$ nucleon), a substantial intensity from nearby corotating interaction regions (see below) competed with the SEP intensity, making the composition data more difficult to interpret. However, [Bucik et al., 2009] reported an iron enhancement at these lower energies on STEREO-A around the middle of 23 May ( $\mathrm{Fe} / \mathrm{O}$ between 0.5 and 1.0) along with an energetic electron event observed by ACE/EPAM, interpreting these as possible flare particles. Though some flare particles could 


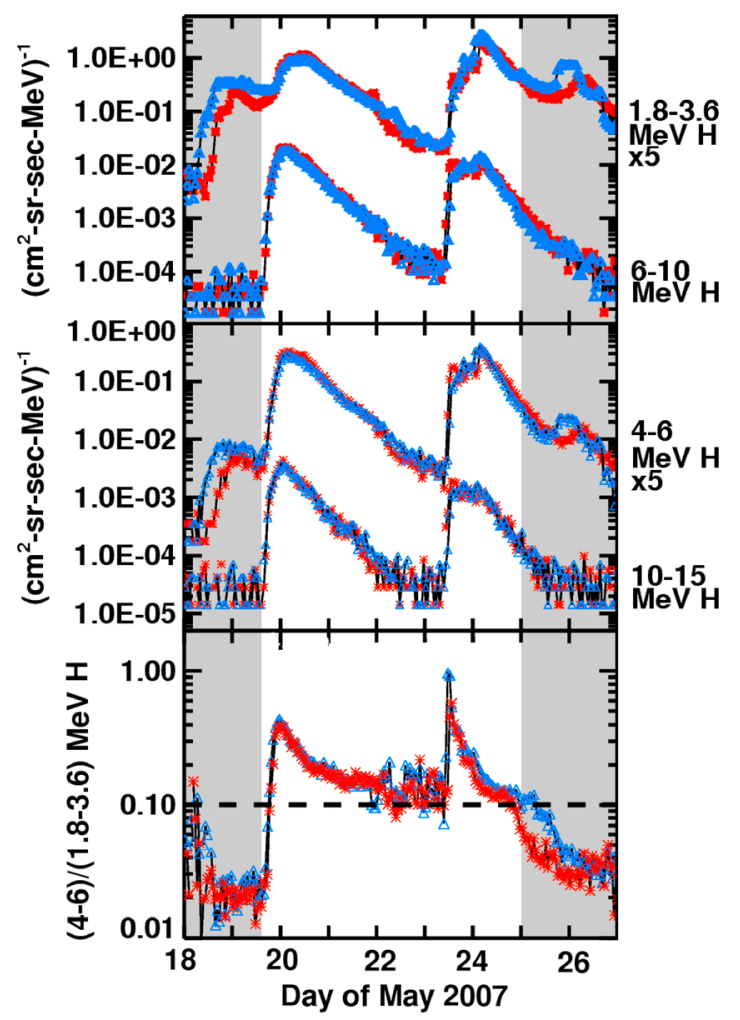

Figure 2. (top and middle) LET data for the entire time range being considered, including the preceding and trailing CIR events (shaded). Four energy ranges (labeled on the right) with the blue triangles for data from the Behind spacecraft and red stars for the Ahead spacecraft. (bottom) The 4-6 to $1.8-3.6 \mathrm{MeV}$ proton intensity ratio, which is an approximate discriminator for CIR versus SEP material from [Leske et al., 2008, 2009]. Values above 0.1 (marked by the dashed line) indicate SEP material, while values below that indicate CIR material.

conceivably have been contributing to the higher-energy LET observations, the LET composition suggests only CMEaccelerated particles were present above $2 \mathrm{MeV} /$ nucleon in the time interval selected for study.

[10] Figure 2 shows proton time profiles for four LET energy ranges between 2 and $15 \mathrm{MeV}$ over the entire time range, from 18 to 27 May. The two SEP events were preceded and trailed by energetic particle events associated with corotating interaction regions (CIRs), in which particles are accelerated by shocks that form when high-speed solar wind streams impact slower-moving streams [GómezHerrero et al., 2009]. SEP events have been shown to have much harder spectra than CIR events, with CIR material tending to have ratios of 4-6 to $1.8-3.6 \mathrm{MeV}$ protons below 0.1 [Leske et al., 2008]. This ratio is plotted in Figure 2 (bottom), and we also note that these CIR events were identified in the surveys of Mason et al. [2009, 2008]. Compositionally, $\mathrm{He} / \mathrm{H}$ ratios of 0.01 or below tend to be observed in SEP events [Leske et al., 2008], and the LET He/H ratio is at or below 0.01 at all energies between 19 and 25 May. We have defined the SEP event interval as beginning at 1500 UT on the 19 May (DOY 139) and ending at 0000 UT on the 25 May (DOY 145), a time range which excludes most of the CIR contribution in the LET energy ranges and agrees with other analyses of these events [Bucik et al., 2009]. The time range which is dominated by CIR material is shaded in Figure 2.

[11] The observed onset times and time-intensity profiles of the earliest arriving LET protons in the 23 May SEP event differed substantially between the two STEREO spacecraft, while those in the 19 May event were almost identical. Figure 3 shows the 19 May (Figure 3, left) and 23 May (Figure 3, right) events, respectively, with the bottom of each indicating the ratio of intensities between STEREO-A and -B. On 19 May, the time-intensity profiles from A and B largely tracked each other at all energies and lacked significant intensity fluctuations. The intensities of 6-10 MeV protons between A and $\mathrm{B}$ never differed by more than a factor of 2 at any time during the event. On 23 May, the energetic particle event began around 1000 UT on A but not until an hour later on B, and the two time profiles differed by more than a factor of 2 until 1400 UT. A $1 \mathrm{~h}$ difference in arrival time is unusually large, since it is equivalent to the travel time from the Sun to $1 \mathrm{AU}$ for a $10 \mathrm{MeV}$ proton. After $1400 \mathrm{UT}$, the intensities differed qualitatively in their short timescale fluctuations, as evidenced by the greater deviation in the ratio of LET-A to LET-B from 1 than in the previous event.

[12] The anisotropies seen by LET followed the same pattern as the time profiles: STEREO-A and STEREO-B observed similar anisotropies as a function of time during the 19 May event, and dissimilar anisotropies as a function of time during the 23 May event. The first-arriving particles of the 19 May event at both STEREO-A and -B arrived along the measured magnetic field from the Sun, and the particles gradually became more isotropic with time, as is typically observed in small SEP events. Except where noted, all the following observations and analysis are of the 23 May event, which, we will show, behaves atypically. The 19 May event is simply presented here for contrast, as it represents a fairly typical CME-driven SEP event observed at $1 \mathrm{AU}$.

[13] In order to highlight fluctuations away from a typical time-intensity profile for an SEP event, we have created an "adjusted intensity" by subtracting out the overall shape of the time-intensity profile. The adjusted intensity was found by taking the best fit of the profile to the function,

$$
F(t)=\frac{P_{1} t^{P_{2}}}{e^{P_{3} t}-1}
$$

where $P_{1}, P_{2}$ and $P_{3}$ are fitted constants, and then subtracting off this fit. This profile was chosen largely empirically, with exponential falloffs being commonly observed in SEP events [Mason et al., 2006] and a power law expected in the earliest-arriving particles from a simple ballistic model. The resulting adjusted intensity is the difference between the profile fit and the measured intensity (measured intensity minus profile). This model fits observed smooth time-intensity profiles like the one on 19 May very well (see Figure 4). The adjusted intensity for the 23 May event is fit from 1200 UT 23 May to 1200 UT 24 May, and the resulting adjusted intensity values (Figure 5) are 3-4 times higher than those for the 19 May event in Figure 4, indicating substantially more fluctuation in the 23 May event. 


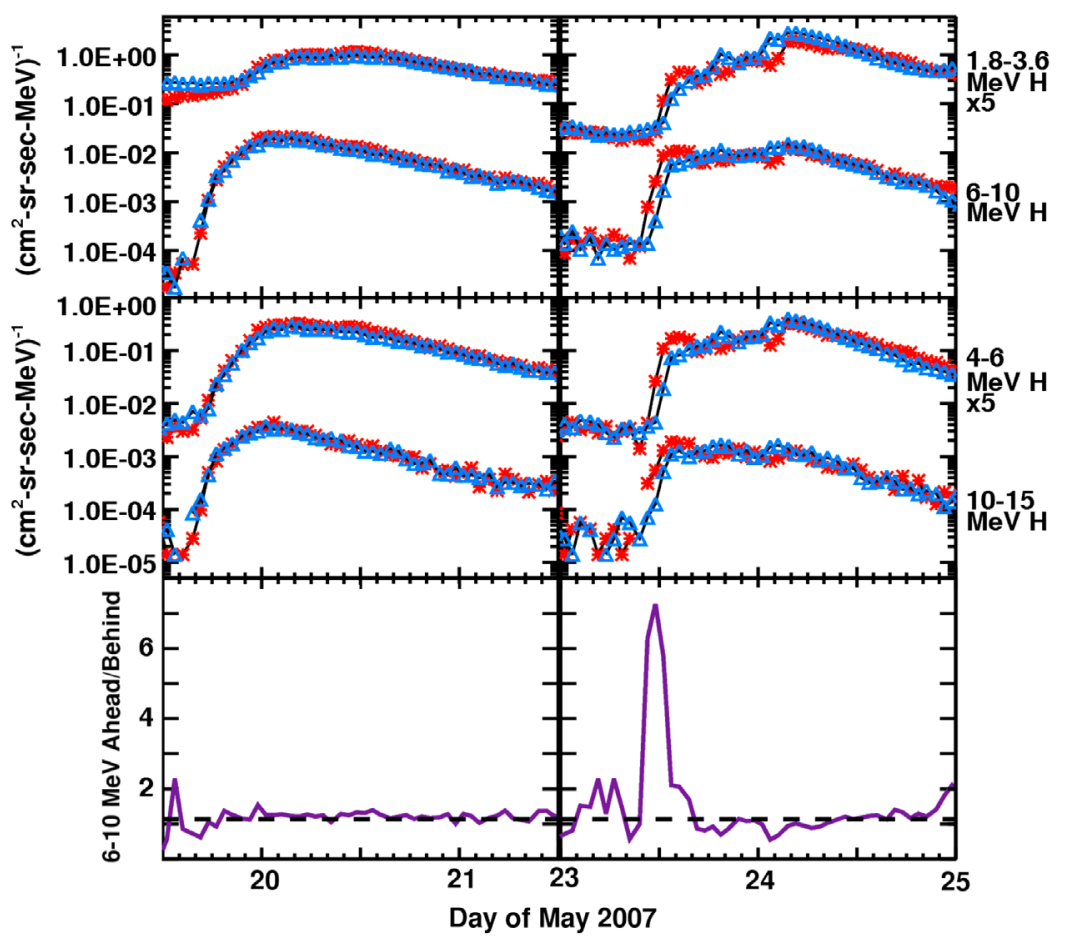

Figure 3. (top and middle) LET data comparison of the (left) 19 May and (right) 23 May events. (bottom) The ratio of the intensity at Ahead over the intensity at Behind for 6-10 MeV protons. In the 19 May event, the SEP event begins simultaneously at both spacecraft, and the time profiles match very closely (the ratio of Ahead to Behind stays near 1). The SEP event begins $\sim 1 \mathrm{~h}$ earlier at Ahead than Behind in the 23 May event, and their time profiles around the flare peak are substantially different. This difference is reflected in the ratio of Ahead to Behind, which differs by a factor of 7 during the rising phase and varies from 1 around the peak.

[14] Figure 5 shows examples of the measured angular distributions of LET-A and LET-B 4 to $6 \mathrm{MeV}$ protons during the 23 May SEP event, along with the adjusted intensity. These data are in RTN coordinates, where R points radially away from the Sun, $\mathrm{T}$ is perpendicular to $\mathrm{R}$ and lies in the ecliptic plane, and $\mathrm{N}$ completes the right-handed set. The $0^{\circ}$ lies along the $\mathrm{R}$ direction, with the angle increasing counterclockwise, as indicated on the plot. The vertical lines

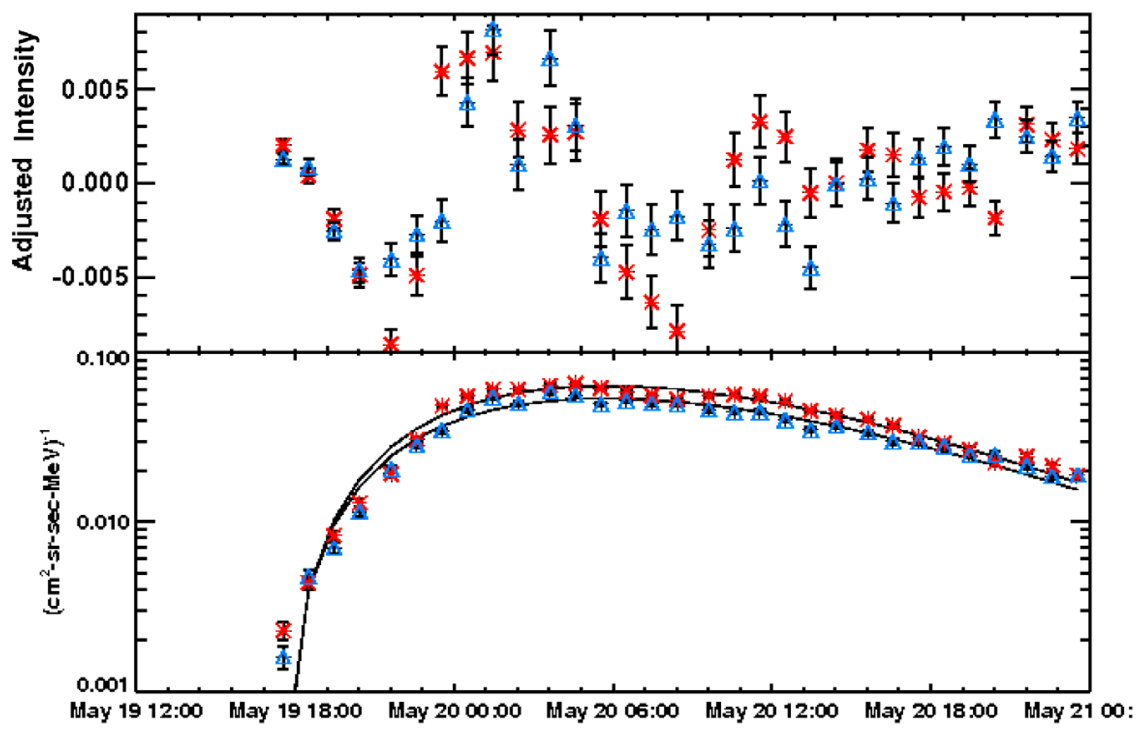

Figure 4. (top) Adjusted intensity equal to the difference between the measured intensity and (bottom) profile fit given in equation (1) for 4-6 MeV protons. This profile is for the 19 May event and demonstrates how closely the functional form fits the actual data for the 19 May event, a typical small SEP event. 

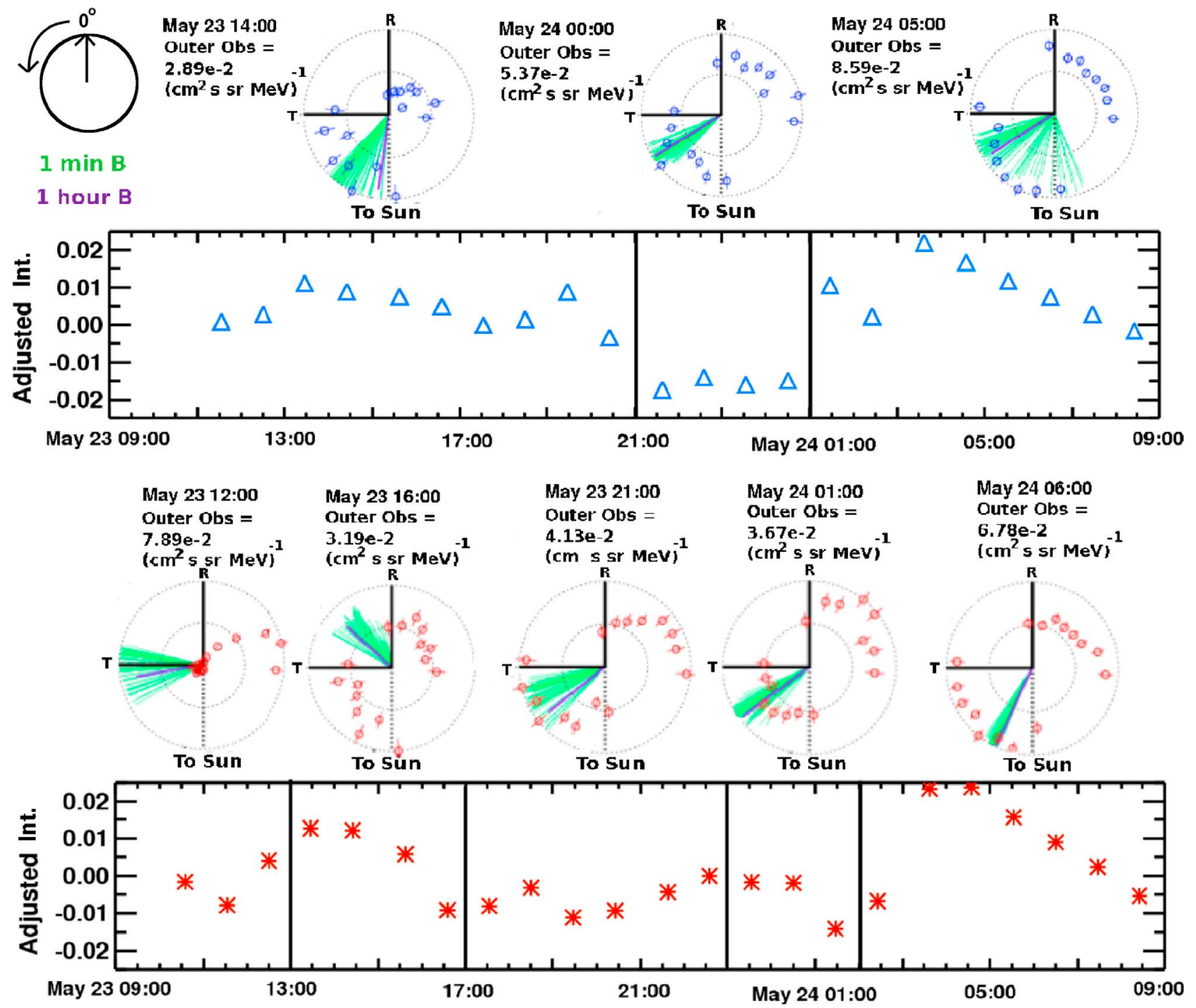

Figure 5. Anisotropies and 4-6 MeV LET intensities for the 23 May event for (top) STEREO-B and (bottom) STEREO-A. The circular plots are hour snapshots of the R-T plane, which LET observes, with the thick purple line being the hour-long average of the magnetic field direction and the green thin lines being the 1 min observations. The circular points indicate the particle intensity in a given look direction, with the absolute intensity at the outer ring indicated next to it. The vertical lines on the adjusted intensity plots are chosen at the times when the particle anisotropy changes significantly, with each section having a representative anisotropy plot above it. The changes in the character of the anisotropies correspond roughly with the changes in the adjusted intensity (the difference in intensity between the absolute intensity in Figure 4 and the profile fit) in each spacecraft and do not correspond between the two spacecraft. The error bars on the adjusted intensity points are smaller than the points in this scale.

on the intensity plot are chosen where the characteristics of the anisotropy distribution change significantly, e.g., from sunward-flowing particles to antisunward flowing particles. STEREO-B observed a magnetic field which was roughly along the Parker spiral angle $\left(135^{\circ}\right)$ during the entire 23 May event except for a brief excursion out of the ecliptic plane (in the $\mathrm{N}$ direction, not shown) between 2100 UT 23 May and 0100 UT 24 May. The particles at STEREO-B flowed antisunward at the beginning and end of the event, that is, they have their greatest intensity in the sunward side of the detector. Between 2100 UT 23 May and 0100 UT 24 May the particles became isotropic, appearing at all angles in the
$\mathrm{R}-\mathrm{T}$ plane roughly equally. The isotropy was reflected in the drop in the adjusted intensity, as more of the particles were outside of LET's field of view and thus did not show up in the measured intensity. ACE/EPAM observed bi-directional streaming of $\sim 1 \mathrm{MeV}$ protons for $\sim 3.5 \mathrm{~h}$ around the peak of the event, but otherwise STEREO-B and ACE/EPAM observed proton pitch angle distributions which were fairly reflective of a simple Parker spiral magnetic field connection to a particle source close to the Sun.

[15] The changes in anisotropy and magnetic field angle were more complex at STEREO-A. During the first $3 \mathrm{~h}$ of the 23 May event, the magnetic field line was $\sim 90^{\circ}$ from the 

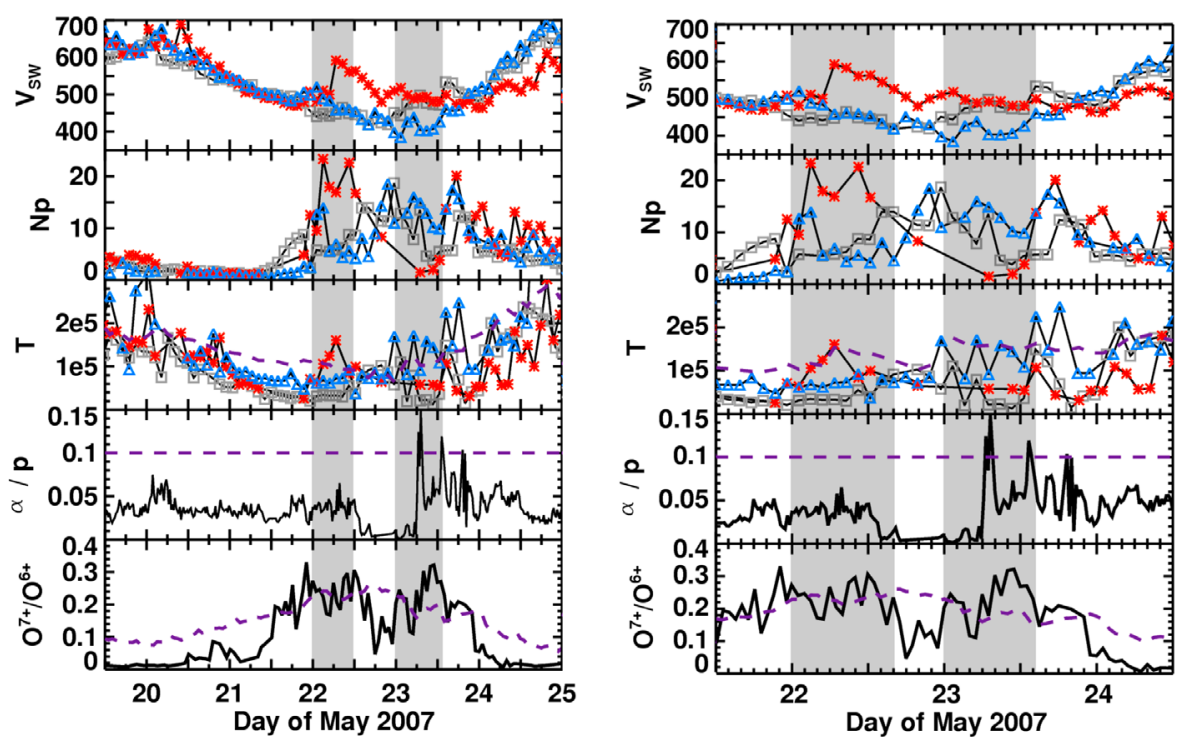

Figure 6. Solar wind data for (left) the entire SEP period and (right) just during the passage of the ICMEs. The red stars are for STEREO-A, blue triangles are for STEREO-B, and the gray squares are for ACE, with the bottom two plots solely ACE data. The shaded regions represent the boundaries of flux ropes according to Liu et al. [2008] and Kilpua et al. [2009]. From top to bottom, the solar wind speed, density, temperature, the $\alpha$ to proton ratio, and the solar wind $\mathrm{O}^{7+} / \mathrm{O}^{6+}$ ratio. The dashed purple lines in the bottom three panels mark the thresholds for ICMEs described in the text, with the line in the temperature plot derived for STEREO-A.

Earth-Sun line, and it appears as if the first particles entered LET from the back (at $\sim 280^{\circ}$ ) rather than from the front. The field line then shifted to $\sim 45^{\circ}$, and the particle distribution became fairly isotropic. Starting at $1700 \mathrm{UT}$, the field turned to roughly the Parker spiral angle, and the particles became bidirectional, then shifted to primarily sunward at 2300 UT and to primarily antisunward at 0200 UT 24 May. The shift in field angle at $1700 \mathrm{UT}$ and the reversal of the proton direction at 0200 UT 24 May were accompanied by a large decrease and increase in the adjusted intensity, respectively, while the anisotropy and field angle change at 1300 UT was accompanied by a smaller increase in the adjusted intensity. The 2300 UT change was the only pitch angle and field angle change with no obvious change in the adjusted intensity. The intensity fluctuations around the peak therefore were loosely correlated with changes in the character of the proton pitch angle distribution.

[16] Figure 6 presents an overview of solar wind data from ACE and STEREO for the entire time range of the energetic particle events (Figure 6, left) and for the subset of time during the 23 May event (Figure 6, right). Though no one signature is definitive, ICME material can be identified in situ through a combination of several plasma, particle and magnetic field characteristics. Plasma signatures include abnormally low proton temperatures [Gosling et al., 1973], enhancements in helium [Hirshberg et al., 1972] and enhancements in $\mathrm{O}^{7+} / \mathrm{O}^{6+}$ [Zurbuchen and Richardson, 2006]. The plasma, magnetic field and suprathermal electron data in Figure 6 show that from 21 to 24 May, the solar wind plasma had a complex structure due to the presence of ICMEs near or inside the heliospheric current sheet. The dashed purple lines denote the expected values in the solar wind, where values above this line indicate the likely presence of
ICME material [Richardson and Cane, 2004]. The temperatures observed by STEREO-A and ACE on 23 May and STEREO-B and ACE on 22 May are low compared to those expected for solar wind of this velocity [Elliott et al., 2005]. ACE observed intermittent enhancements in $\alpha / p$ above typical solar wind values [Hirshberg et al., 1972] on 23 May and in $\mathrm{O}^{7+} / \mathrm{O}^{6+}$ above solar wind values on 22 and 23 May, suggesting ICME material was almost certainly present during those times.

[17] ICMEs often are observed to contain magnetic flux ropes, marked by a field magnitude enhancement and smooth rotation of the field [Burlaga, 1988]. Because magnetic field lines that make up the flux rope and other portions of the ICME are often connected to the Sun at both ends, particles can be injected on both ends, leading to bidirectional pitch angle distributions (most notably in the suprathermal electrons [Gosling et al., 1987]). Figure 7 shows the suprathermal electron pitch angle and magnetic field data from STEREO-B, ACE and STEREO-A in RTN coordinates. Though bidirectional streaming is sometimes observed in the trailing part of CIRs like the one immediately preceding the SEP events [Lavraud et al., 2010], the intermittent bidirectional streaming in the suprathermal electrons from halfway through 21 May until the CIR appears on 25 May suggests that ICMEs are present, perhaps multiple CMEs. Five time periods which exhibit field enhancements and rotations characteristic of ICME flux ropes are denoted by arrows and numbered in Figure 7. We note that a single ICME may be made up of multiple periods, as ICMEs can be complex structures with multipart flux ropes.

[18] The presence of ICMEs during this period is supported by previous analyses in the literature. Liu et al. [2008] reported on an ICME flux rope seen by both STEREO 

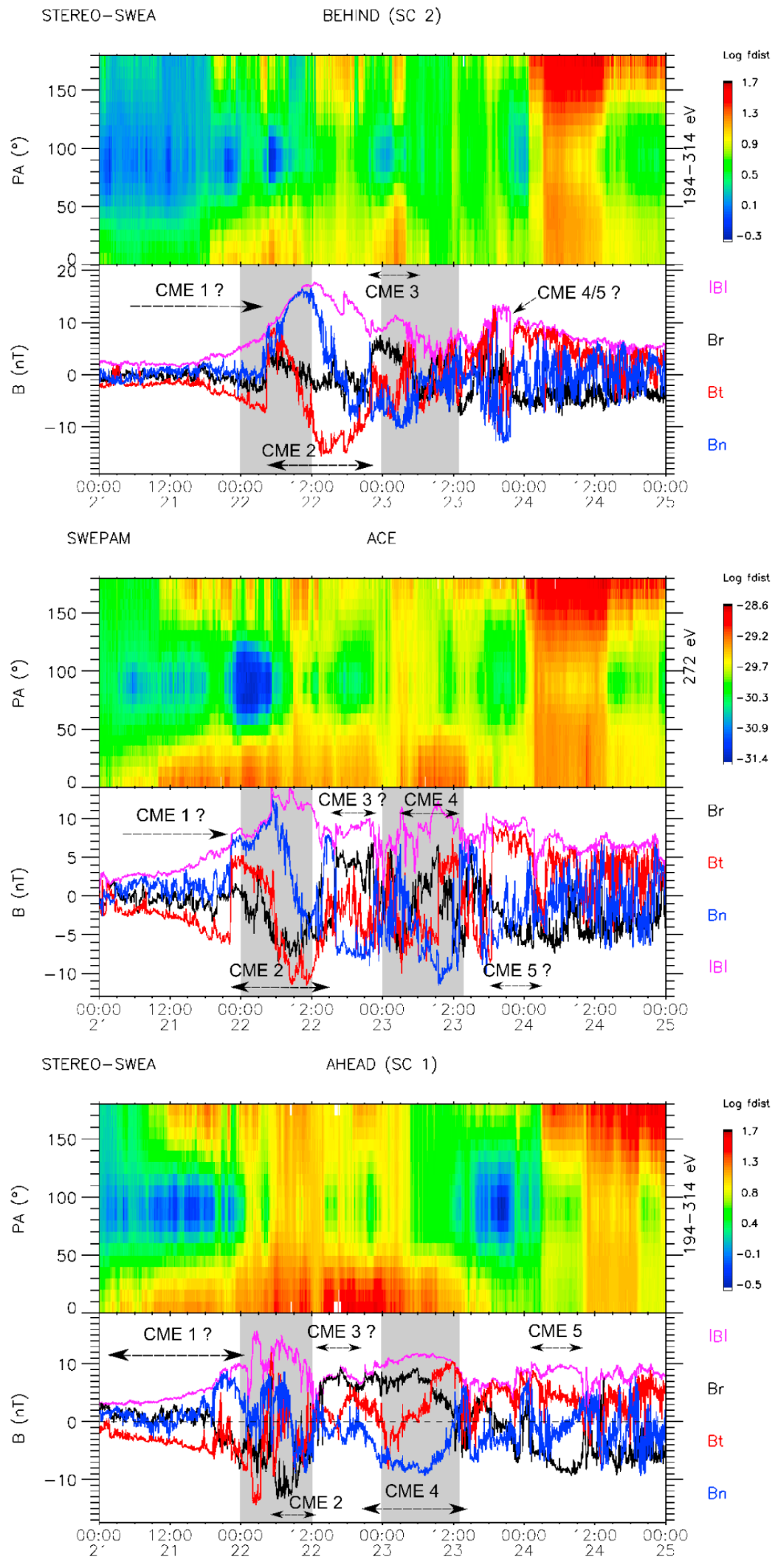

Figure 7. Suprathermal electron pitch angles and magnetic field components from (top) STEREO-B, (middle) ACE, and (bottom) STEREO-A. The $0^{\circ}$ pitch angles are parallel to the field, and the $180^{\circ}$ pitch angles are antiparallel to the field. Bidirectional intervals have roughly equal intensities at $0^{\circ}$ and $180^{\circ}$. Possible flux ropes identified by magnetic field signatures are marked, and the shaded regions represent the boundaries of flux ropes according to Liu et al. [2008] and Kilpua et al. [2009]. 


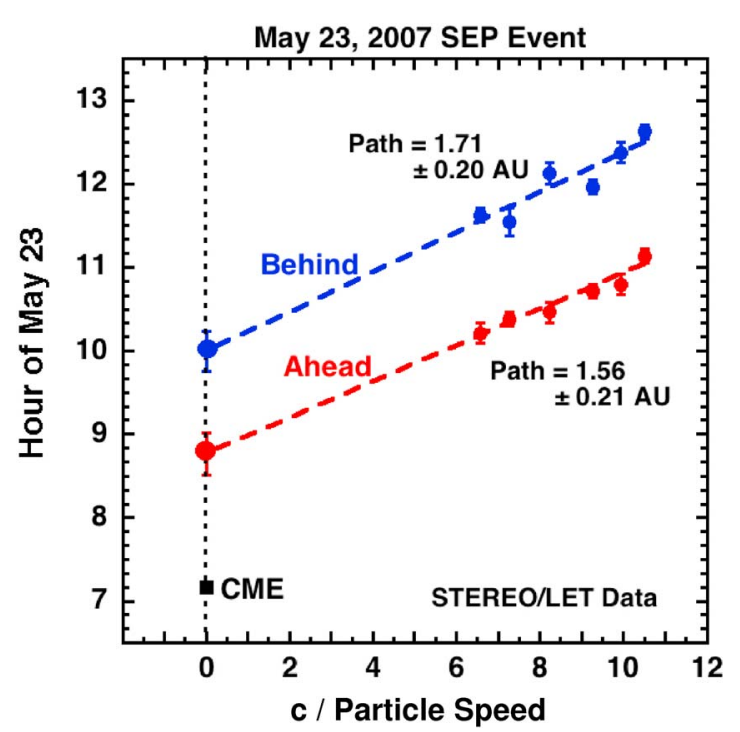

Figure 8. STEREO/LET energetic proton onset times at each energy versus the speed of light over the particle speed for the 23 May event. The dashed lines are least square fits that, when extrapolated to $c / v=0$, provide a measure of when the particles were accelerated at the Sun. The slopes of these lines indicate the travel distance of the particles.

spacecraft, ACE and Wind on 22 May, corresponding to period 2 in Figure 7 and marked with a gray box in Figure 6. They performed a Grad-Shafranov reconstruction of the flux rope geometry, finding a flattened flux rope with a diameter of roughly $0.15 \mathrm{AU}$ which was highly inclined to the plane containing the spacecraft. They concluded that STEREO-B passed close to the axis of the flux rope while STEREO-A only grazed the flank, with ACE in between, and that the flux rope was completely past the spacecraft before the energetic particle event on 23 May. Kilpua et al. [2009] analyzed the plasma data during this time period in detail and found that STEREO-B and Wind (near ACE at L1) observed a magnetic cloud on 22 May, agreeing with Liu et al. [2008]. However, they also argued that STEREO A observed another flux rope on the first half of 23 May, marked in Figure 6 with shaded regions and corresponding to period 4 in Figure 7. The STEREO heliospheric imager catalog (http://www.sstd. rl.ac.uk/stereo/CMELIST 2007.xls) lists multiple ICMEs visible in the interplanetary medium in the days before this event. The ACE (http://www-ssc.igpp.ucla.edu/ jlan/ACE/Level3/ ICME_List_from_Lan_Jian.pdf) and STEREO (http://wwwssc.igpp.ucla.edu/ jlan/STEREO/Level3/STEREO Level3_ICME.pdf) ICME lists state that STEREO-B and ACE are embedded in ICME material on 22 May, emerging at 2200 UT and 1340 UT, respectively, while STEREO-A remains in ICME material until 1330 UT 23 May. We concur with these previous analyses and conclude that at least two ICMEs are near the Earth during 22 and 23 May, and we suggest that there may be up to five or that the ICMEs are complex, multipart structures.

[19] Closer examination of the suprathermal electron data in Figure 7 shows the overall structure of this interval is very complex. The more intense, relatively isotropic enhancement in the suprathermal electrons on 24 May is nonadia- batic heating often seen in the vicinity of CIRs [Lavraud et al., 2010]. Since the suprathermal electron strahl always flows away from the Sun, a switch in the direction relative to the magnetic polarity indicates a crossing of the heliospheric current sheet from one sector to another [Kahler and Lin, 1994]. Though the location cannot be determined precisely due to the complexity of this time interval, on 18 May the electron strahl is at 0 degrees and by 25 May it had switched to 180 degrees, indicating the spacecraft crossed a current sheet. ICMEs are often present in and around CIRs because they are produced in and around the streamer belt [Rouillard et al., 2009], and ICMEs embedded in sector boundaries tend to introduce a high degree of variability [Crooker et al., 1999]. We conclude that this SEP event occurs during a complex interval, with multiple ICMEs in a CIR near a sector boundary and different spacecraft simultaneously embedded in different plasma structures.

\section{Analysis}

[20] The differences in the 23 May energetic particle event observed by the three spacecraft can be attributed to the spacecrafts' locations in different plasma structures, even though they are only separated by $\sim 5-10^{\circ}$. During the initial arrival of the particles around 1000 UT 23 May, STEREO-A is embedded in an ICME, as suggested by unusual proton anisotropies and magnetic field angle (Figures 5 and 7 ) and ICMElike plasma properties (Figure 6). However, STEREO-B is in the solar wind outside the ICME at that time, as it sees a magnetic field angle close to the Parker spiral with particles coming from the Sun as well as typical solar wind plasma. ACE, which is in between the two STEREO spacecraft, is likely skimming the edge of the ICME, as evidenced by the intermittent spikes in $\alpha$ in Figure 6. As discussed by Gosling et al. [2004a], the foot points of magnetic field lines at the Sun inside and outside ICMEs are widely separated from each other. The exact separation of the foot points of STEREO-A and STEREO-B's field lines cannot be calculated directly without a detailed ICME model, but if we assume the leg of the ICME comes out radially and the topology outside the ICME is a Parker spiral, the difference is of the order of several tens of degrees or more.

[21] For clarity, we divide the event observed by STEREOA into first and second halves, before and after 24 May 2:00. As shown in Figure 1, STEREO-A and -B would be well connected to the active region with a standard Parker spiral connection during this event, and the antisunward streaming protons in the second half of the STEREO-A event and during the majority of the event on STEREO-B probably reflected a simple Parker spiral connection. The first half of the STEREO-A event is the anomalous observation, reflecting the nonstandard connection produced by the spacecraft being inside an ICME. Given the iron enhancement [Bucik et al., 2009] reported to be observed by SIT-A, the LET-A particles in the first half of the event may have included some flare-accelerated particles traveling through the ICME rather than solely the CME-accelerated particles that STEREO-B observed.

[22] The LET protons arriving $\sim 1 \mathrm{~h}$ early at STEREO-A reflect the difference in source connection between STEREO$\mathrm{A}$ and $-\mathrm{B}$. Figure 8 presents the arrival times at each spacecraft of the earliest-arriving particles as a function of their 
inverse speed, from which the particle travel distance and injection time can be inferred. The nominal Parker spiral travel distance is only 1.2 AU, so the measured distances are somewhat larger than would be expected for the solar wind, but shorter than the 2-3 AU distances typically observed in ICMEs [Larson et al., 1997]. The measured distances are comparable between the two spacecraft. In this type of plot, increased particle scattering during transport manifests as an increase in the measured travel distance, so the extra $0.5 \mathrm{AU}$ travel distance above the Parker spiral distance seen by STEREO-B can likely be attributed to particle scattering [Chollet et al., 2007]. However, the smoothness of the magnetic field inside ICMEs [Torsti et al., 2004] means the particles observed by STEREO-A were likely scatter-free. The fit indicates that the particles observed by STEREO-A were injected $\sim 1 \mathrm{~h}$ before those observed by STEREO-B. Since the magnetic topology inside ICMEs is likely to be very different from outside, the particle acceleration conditions are likely to be different as well, so the particle acceleration may have begun earlier at the location to which STEREO-A connected. Thus, a magnetic connection to a different location could naturally produce the particles arriving $\sim 1$ h later at STEREO-B.

[23] The field of view of the LET instrument may play a role in producing the intensity fluctuations seen in both STEREO spacecraft. von Rosenvinge et al. [2009] discussed how, in ICMEs, the field line is frequently out of the viewing cone of STEREO-LET, which is at a fixed angle. As a field line moves to high angles relative to the ecliptic, it will flap in and out of the LET field of view producing fluctuations in the particle intensity if there is an anisotropic angular distribution and most of the particles are not seen by the instrument. The major dip in STEREO B adjusted intensity (Figure 5) between 2100 UT 23 May and 0100 UT 24 May is just such a fluctuation, as can be seen by the large change in magnetic field angle at this time in Figure 7 (top).

[24] However, STEREO-A probably has some fluctuations produced by small changes in connection point rather than the instrument field of view. Both halves of the event, the first half with significant particle intensity fluctuations and the second half with only small fluctuations, show roughly the same amount of fluctuation in the magnetic field angle, suggesting that field lines should not be flapping out of LET's field of view any more during the first half than the second. This contrasts with the fluctuation in STEREO-B, where the character of the magnetic field changes dramatically when the particle intensity changes, and it suggests that the fluctuations in STEREO-A may have another explanation. Discontinuous intensity changes in ions and electrons have been linked to a spacecraft observing patches of field lines which connect to different portions of the same event at the Sun [Chollet et al., 2009]. Coronal dimmings, which have been associated with the foot points of CMEs [Thompson et al., 2000], have size scales on the order of 10 degrees [Harrison and Lyons, 2000], so the particle acceleration region should be roughly on this scale. If the particle source region is inhomogeneous on that scale, it could produce the intensity fluctuations seen here, as the spacecraft connects to different parts of the CME foot point region.

[25] The contrast between the 23 May event and the 19 May event illustrates the benefits of using multiple spacecraft and multiple instruments to determine magnetic geome- try from energetic particle measurements. In STEREO-B, the LET time-intensity profiles for the two events are fairly similar to each other, so if observed from only this spacecraft, the 23 May event would probably be interpreted as unremarkable and very similar to the 19 May event. Including STEREO-A observations, even though the two spacecraft were separated by less than $10^{\circ}$, reveals a complicated ICME structure that STEREO-B narrowly missed. Including the suprathermal electron and magnetic field observations allows interpretation of the fluctuations that could not be performed without these additional data. As the STEREO spacecraft move further apart, the differences and similarities between events at widely separated spacecraft will reveal even more about the magnetic structure of the inner heliosphere.

\section{Conclusions}

[26] We have presented energetic ion time-intensity profiles for a pair of SEP events on 19 May and 23 May observed by the twin STEREO spacecraft and ACE. The 19 May event was very similar at the two STEREO spacecraft, but the STEREO observations of the 23 May event were complicated by the presence of an ICME during the first part of the STEREO-A event. This ICME caused an observed delay in the arrival of the solar energetic particles at LET-B and significant fluctuations in the particle intensity at LET-A, because the connection point of the field lines was markedly different inside the ICME than outside. Fluctuations related to other shifts in connection point were probably observed by LET-A, though intensity fluctuations due to limits on the LET field of view were likely present in both spacecraft. Though the two STEREO spacecraft were only separated by $\sim 9^{\circ}$, with ACE in between the two, all three had very different magnetic connections during the 23 May event, illustrating how single-spacecraft observations can cause a complex event to be interpreted as a simple one. Multispacecraft observations offer a much clearer picture of the magnetic connections in SEP events.

[27] Acknowledgments. This work was supported by NASA under grants NAS5-03131, NNX08AI11G, NNX07AN45G and by UC Berkeley under contract SA4889-26309. We thank the STEREO/PLASTIC and ACE/SWICS teams for the use of their data.

[28] Philippa Browning thanks Tycho von Rosenvinge and another reviewer for their assistance in evaluating this paper.

\section{References}

Acuña, M. H., D. Curtis, J. L. Scheifele, C. T. Russell, P. Schroeder, A. Szabo, and J. G. Luhmann (2008), The STEREO/IMPACT magnetic field experiment, Space Sci. Rev., 136, 203-226, doi:10.1007/s11214007-9259-2.

Bieber, J. W., J. Clem, P. Evenson, R. Pyle, D. Ruffolo, and A. Saiz (2005), Relativistic solar neutrons and protons on 28 October 2003, Geophys. Res. Lett., 32, L03S02, doi:10.1029/2004GL021492.

Bucik, R., U. Mall, R. Gómez-Herrero, A. Korth, and G. M. Mason (2009), STEREO observations of energetic ions in corotating interaction regions during the May 2007 solar events, Sol. Phys., 259, 361-380, doi:10.1007/ s11207-009-9415-9.

Burlaga, L. F. (1988), Magnetic clouds and force-free fields with constant $\alpha$, J. Geophys. Res., 93(A7), 7217-7224, doi:10.1029/JA093iA07p07217.

Chollet, E. E., and J. Giacalone (2008), Multispacecraft analysis of energetic ion flux dropouts, Astrophys. J., 688, 1368-1373, doi:10.1086/ 592378 .

Chollet, E. E., J. Giacalone, J. E. Mazur, and M. Al Dayeh (2007), A new phenomenon in impulsive-flare-associated energetic particles, Astrophys. J., 669, 615-620, doi:10.1086/521670. 
Chollet, E. E., J. Giacalone, R. M. Skoug, J. T. Steinberg, and J. T. Gosling (2009), Spatial offsets of interplanetary ion and electron source regions, Astrophys. J., 705, 1492-1495, doi:10.1088/0004-637X/705/2/1492.

Crooker, N. U., S. Shodhan, R. J. Forsyth, M. E. Burton, J. T. Gosling, R. J. Fitzenreiter, and R. P. Lepping (1999), Transient aspects of stream interface signatures, in Solar Wind Nine, edited by S. Habbal et al., pp. 597600, Am. Insti. of Phys., New York.

Elliott, H. A., D. J. McComas, N. A. Schwadron, J. T. Gosling, R. M. Skoug, G. Gloeckler, and T. H. Zurbuchen (2005), An improved expected temperature formula for identifying interplanetary coronal mass ejections, J. Geophys. Res., 110, A04103, doi:10.1029/2004JA010794.

Galvin, A. B., et al. (2008), The Plasma and Suprathermal Ion Composition (PLASTIC) investigation on the STEREO observatories, Space Sci. Rev. 136, 437-486, doi:10.1007/s11214-007-9296-x.

Giacalone, J., J. R. Jokipii, and J. E. Mazur (2000), Small-scale gradients and large-scale diffusion of charged particles in the heliospheric magnetic field, Astrophys. J., 532, L75-L78, doi:10.1086/312564.

Gloeckler, G., et al. (1998), Investigation of the composition of solar and interstellar matter using solar wind and pickup ion measurements with SWICS and SWIMS on the ACE spacecraft, Space Sci. Rev., 86, 497539, doi:10.1023/A:1005036131689.

Gold, R. E., S. M. Krimigis, S. E. Hawkins, D. K. Haggerty, D. A. Lohr, E. Fiore, T. P. Armstrong, G. Holland, and L. J. Lanzerotti (1998), Electron, proton, and $\alpha$ monitor on the advanced composition explorer spacecraft, Space Sci. Rev., 86, 541-562, doi:10.1023/A:1005088115759.

Gómez-Herrero, R., A. Klassen, R. Müller-Mellin, B. Heber, R. WimmerSchweingruber, and S. Böttcher (2009), Recurrent CIR-accelerated ions observed by STEREO SEPT, J. Geophys. Res., 114, A05101, doi:10.1029/ 2008JA013755.

Gosling, J. T., V. Pizzo, and S. J. Bame (1973), Anomalously low proton temperatures in the solar wind following interplanetary shock wavesEvidence for magnetic bottles?, J. Geophys. Res., 78(13), 2001-2009, doi:10.1029/JA078i013p02001.

Gosling, J. T., D. N. Baker, S. J. Bame, W. C. Feldman, R. D. Zwickl, and E. J. Smith (1987), Bidirectional solar wind electron heat flux events, J. Geophys. Res., 92(A8), 8519-8535, doi:10.1029/JA092iA08p08519.

Gosling, J. T., R. M. Skoug, D. J. McComas, and J. E. Mazur (2004a), Correlated dispersionless structure in suprathermal electrons and solar energetic ions in the solar wind, Astrophys. J., 614, 412-419, doi:10.1086/ 423368 .

Gosling, J. T., C. A. de Koning, R. M. Skoug, J. T. Steinberg, and D. J. McComas (2004b), Dispersionless modulations in low-energy solar electron bursts and discontinuous changes in the solar wind electron strahl, J. Geophys. Res., 109, A05102, doi:10.1029/2003JA010338.

Harrison, R. A., and M. Lyons (2000), A spectroscopic study of corona dimming associated with coronal mass ejection, Astron. Astrophys. 358, 1097-1108.

Hirshberg, J., S. J. Bame, and D. E. Robbins (1972), Solar flares and solar wind helium enrichments: July 1965-July 1967, Sol. Phys., 23, 467-486, doi:10.1007/BF00148109.

Kahler, S. (1994), Injection profiles of solar energetic particles as functions of coronal mass ejection heights, Astrophys. J., 428, 837-842, doi:10.1086/174292.

Kahler, S., and R. P. Lin (1994), The determination of interplanetary magnetic field polarities around sector boundaries using $\mathrm{E}>2 \mathrm{keV}$ electrons, Geophys. Res. Lett., 21(15), 1575-1578, doi:10.1029/94GL01362.

Kaiser, M. L., T. A. Kucera, J. M. Davila, O. C. St. Cyr, M. Guhathakurta, and E. Christian (2008), The STEREO Mission: An introduction, Space Sci. Rev., 136, 5-16, doi:10.1007/s11214-007-9277-0.

Kallenrode, M.-B. (1993), Neutral lines and azimuthal "transport" of solar energetic particles, J. Geophys. Res., 98(A4), 5573-5591, doi:10.1029/ 92JA02778.

Kilpua, E. K. J., et al. (2009), Multispacecraft observations of magnetic clouds and their solar origins between 19 and 23 May 2007, Sol. Phys. 254, 325-344, doi:10.1007/s11207-008-9300-y.

Lario, D., M.-B. Kallenrode, R. B. Decker, E. C. Roelof, S. M. Krimigis, A. Aran, and B. Sanahuja (2006), Radial and longitudinal dependence of solar 4-13 MeV and 27-37 MeV proton peak intensities and fluences: Helios and Imp 8 observations, Astrophys. J., 653, 1531-1544, doi:10.1086/508982

Larson, D. E., et al. (1997), Tracing the topology of the October 18-20, 1995 magnetic cloud with $\sim 0.1-10^{2} \mathrm{keV}$ electrons, Geophys. Res. Lett., 24(15), 1911-1914, doi:10.1029/97GL01878.

Lavraud, B., et al. (2010), Statistics of counterstreaming solar wind suprathermal electrons at solar minimum: STEREO observations, Ann. Geophys. 28, 233-246, doi:10.5194/angeo-28-233-2010.

Leske, R. A., et al. (2008), Observations of corotating interaction regions from STEREO and ACE, in Proceedings of the 30th International Cosmic Ray Conference. July 3-11, 2007, Mérida, Yucatán, Mexico, vol. 1, edited by R. Caballero et al., pp. 375-378, Univ. Nac. Autó. de México, Mexico City.

Leske, R. A., G. M. Mason, R. A. Mewaldt, C. M. S. Cohen, A. C. Cummings, A. W. Labrador, E. C. Stone, M. E. Wiedenbeck, and T. T. von Rosenvinge (2009), Multi-point observations of corotating interaction regions from STEREO and ACE, paper presented at 31st International Cosmic Ray Conference, Lodz, Poland, 7-15 July.

Liu, Y., J. G. Luhmann, K. E. J. Huttunen, R. P. Lin, S. D. Bale, C. T. Russell, and A. B. Galvin (2008), Reconstruction of the 2007 May 22 magnetic cloud: How much can we trust the flux-rope geometry of CMEs?, Astrophys. J., 677, L133-L136, doi:10.1086/587839.

Luhmann, J. G., et al. (2008), STEREO IMPACT investigations, goals, measurements and data products overview, Space Sci. Rev., 136, 117-184, doi:10.1007/s11214-007-9170-x.

Mason, G. M., et al. (2002), Spectral properties of He and heavy ions in ${ }^{3} \mathrm{He}-$ rich solar flares, Astrophys. J., 574, 1039-1058, doi:10.1086/ 341112 .

Mason, G. M., J. E. Mazur, J. R. Dwyer, J. R. Jokipii, R. E. Gold, and S. M. Krimigis (2004), Abundances of heavy and ultraheavy ions in ${ }^{3} \mathrm{He}$-rich solar flares, Astrophys. J., 606, 555-564, doi:10.1086/382864.

Mason, G. M., M. I. Desai, C. M. S. Cohen, R. A. Mewaldt, E. C. Stone, and J. R. Dwyer (2006), The role of interplanetary scattering in western hemisphere large solar energetic particle events, Astrophys. J., 647, L65-L68, doi:10.1086/507469.

Mason, G. M., R. A. Leske, M. I. Desai, C. M. S. Cohen, J. R. Dwyer, J. E. Mazur, R. A. Mewaldt, R. E. Gold, and S. M. Krimigis (2008), Abundances and energy spectra of corotating interaction region heavy ions observed during solar cycle 23, Astrophys. J., 678, 1458-1470, doi:10.1086/533524.

Mason, G. M., M. I. Desai, U. Mall, A. Korth, R. Bucik, T. T. von Rosenvinge, and K. D. Simunac (2009), In situ observation of CIRs on STEREO, wind, and ACE during 2007-2008, Sol. Phys., 256, $393-$ 408, doi:10.1007/s11207-009-9367-0.

Mazur, J. E., G. M. Mason, J. R. Dwyer, J. Giacalone, J. R. Jokipii, and E. C. Stone (2000), Interplanetary magnetic field line mixing deduced from impulsive solar flare particles, Astrophys. J., 532, L79-L82, doi:10.1086/312561.

McComas, D. J., S. J. Bame, P. Barker, W. C. Feldman, J. L. Phillips, P. Riley, and J. W. Griffee (1998), Solar Wind Electron Proton Alpha Monitor (SWEPAM) for the advanced composition explorer, Space Sci. Rev., 86, 563-612, doi:10.1023/A:1005040232597.

Mewaldt, R. A., et al. (2003), Heavy ion and electron release times in solar particle events, in Proceedings of the 28th International Cosmic Ray Conference. July 31-August 7, 2003, Trukuba, Japan, edited by T. Kajita et al., pp. 3313-3316, Universal Acad. Press, Tokyo.

Mewaldt, R. A., et al. (2008), The Low-Energy Telescope (LET) and SEP central electronics for the STEREO mission, Space Sci. Rev., 136, 285-362, doi:10.1007/s11214-007-9288-x.

Reames, D. V. (2009), Solar release times of energetic particles in groundlevel events, Astrophys. J., 693, 812-821, doi:10.1088/0004-637X/693/ $1 / 812$.

Richardson, I. G., and H. V. Cane (2004), Identification of interplanetary coronal mass ejections at $1 \mathrm{AU}$ using multiple solar wind plasma composition anomalies, J. Geophys. Res., 109, A09104, doi:10.1029/ 2004JA010598.

Rouillard, A. P., et al. (2009), A multispacecraft analysis of a small-scale transient entrained by solar wind streams, Sol. Phys., 256, 307-326, doi:10.1007/s11207-009-9329-6.

Saiz, A., D. Ruffolo, J. W. Bieber, P. Evenson, and R. Pyle (2008), Anisotropy signatures of solar energetic particle transport in a closed interplanetary magnetic field loop, Astrophys. J., 672, 650-658, doi:10.1086/ 523663.

Sauvaud, J.-A., et al. (2008), The IMPACT Solar Wind Electron Analyzer (SWEA), Space Sci. Rev., 136, 227-239, doi:10.1007/s11214-0079174-6.

Smith, C. W., J. L'heureux, N. F. Ness, M. H. Acuña, L. F. Burlaga, and J. Scheifele (1998), The ACE magnetic fields experiment, Space Sci. Rev., 86, 613-632, doi:10.1023/A:1005092216668.

Stone, E. C., A. M. Frandsen, R. A. Mewaldt, E. R. Christian, D. Margolies, J. F. Ormes, and F. Snow (1998), The advanced composition explorer, Space Sci. Rev., 86, 1-22, doi:10.1023/A:1005082526237.

Thompson, B. J., E. W. Cliver, N. Nitta, C. Delannée, and J.-P. Delaboudinère (2000), Coronal dimmings and energetic CMEs in April-May 1998 Geophys. Res. Lett., 27(10), 1431-1434, doi:10.1029/1999GL003668.

Torsti, J., E. Riihonen, and L. Kocharov (2004), The 1998 May 2-3 magnetic cloud: An interplanetary "highway" for solar energetic particles observed with SOHO/ERNE, Astrophys. J., 600, L83-L86, doi: $10.1086 / 381575$. 
Tylka, A. J., C. M. S. Cohen, W. F. Dietrich, S. Krucker, R. E. McGuire, R. A. Mewaldt, C. K. Ng, D. V. Reames, and G. H. Share (2003), Onsets and release times in solar particle events, in Proceedings of the 28th International Cosmic Ray Conference. July 31-August 7, 2003, Trukuba Japan, edited by T. Kajita et al., p. 3305, Universal Acad. Press, Tokyo. von Rosenvinge, T. T., I. G. Richardson, D. V. Reames, C. M. S. Cohen, A. C. Cummings, R. A. Leske, R. A. Mewaldt, E. C. Stone, and M. E. Wiedenbeck (2009), The solar energetic particle event of 14 December 2006, Sol. Phys., 256, 443-462, doi:10.1007/s11207-009-9353-6.

Zurbuchen, T. H., and I. G. Richardson (2006), In-situ solar wind and magnetic field signatures of interplanetary coronal mass ejections, Space Sci. Rev., 123, 31-43, doi:10.1007/s11214-006-9010-4.

E. E. Chollet, A. C. Cummings, R. A. Leske, and R. A. Mewaldt, Space Radiation Laboratory, California Institute of Technology, $1200 \mathrm{E}$.
California Blvd., MC 290-17, Pasadena, CA 91125, USA. (echollet@srl. caltech.edu)

J. T. Gosling, Laboratory for Atmospheric and Space Physics, University of Colorado at Boulder, 1234 Innovation Dr., Boulder, CO 80303, USA.

D. K. Haggerty and E. C. Roelof, Applied Physics Laboratory, Johns Hopkins University, 11100 Johns Hopkins Rd., Laurel, MD 20723, USA.

Q. Hu, Center for Space Plasma and Aeronomy Research, University of Alabama in Huntsville, 320 Sparkman Dr., Huntsville, AL 35899, USA.

D. Larson, Space Sciences Laboratory, University of California, 7 Gauss Way, Berkeley, CA 74720, USA.

B. Lavraud, A. Opitz, and J.-A. Sauvaud, Centre d'Etude Spatiale des Rayonnements, Université de Toulouse, 9 ave. du Colonel Roche, F-31028 Toulouse CEDEX 4, France.

C. T. Russell, Institute of Geophysics and Planetary Physics, University of California, 603 Charles Young Dr. East, Los Angeles, CA 90065, USA. 